\title{
RECEIVED
}

$$
\begin{aligned}
& \text { JUL } 301997 \\
& \text { OSTI SAND--97-1779C } \\
& \text { CONF-9705139-- } 2
\end{aligned}
$$

\section{Magnetically-Excited Flexural Plate Wave Resonator}

\author{
S. J. Martin, M. A. Butler, J. J. Spates*, W. K. Schubert, and M. A. Mitchell \\ Microsensor Research and Development Department \\ Sandia National Laboratories, Albuquerque, NM, USA
}

\section{ABSTRACT}

A flexural plate wave (FPW) resonator was constructed by patterning current lines on a silicon nitride membrane suspended on a rectangular silicon frame. Eigenmodes of the rectangular membrane were excited using Lorentz forces generated between alternating surface currents and a static in-plane magnetic field. The magnetic field strength required for these devices can be achieved with small permanent magnets $\left(\approx 1 \mathrm{~cm}^{3}\right)$. Preferential coupling to a particular membrane mode was achieved by positioning current lines along longitudinal mode antinodes. An equivalent-circuit model was derived that characterizes the input impedance of a one-port device and the transmission response of a two-port device over a range of frequencies near a single membrane resonance. Experiments were performed to characterize the effects of varying magnetic field, ambient gas, gas pressure, and input power. To our knowledge, this is the first experimental demonstration of a resonant FPW device.

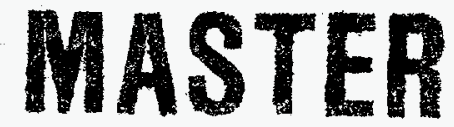

\footnotetext{
*On contract from K-Tech Corp., Albuquerque, NM
} 


\section{DISCLAMMER}

Portions of this document may be illegible in electronic image products. Images are produced from the best available original document. 


\section{INTRODUCTION}

White and Wenzel have shown that flexural plate waves (FPWs) can be excited in a free-standing membrane formed on a silicon substrate ${ }^{1,2}$. Included in the layers of their composite membrane are a piezoelectric $\mathrm{ZnO}$ layer and interdigital transducers that are used to excite the FPWs. In an alternative approach, Giesler and Meyer have shown that electrostatic excitation can also be used to excite these waves ${ }^{3}$. Exciting waves in a thin membrane, as opposed to a thick substrate as in a SAW device, results in a lower operating frequency and, consequently, simpler oscillator electronics. In addition, the confinement of acoustic energy in a thin membrane makes the wave velocity more sensitive to surface perturbations, e.g., accumulation of mass. This results in lower detection limits in sensing applications $^{1}$.

In this paper we describe a new type of FPW device that uses meander-line current conductors patterned on the membrane surface, in combination with a static in-plane magnetic field, to excite and detect flexural plate waves. These waves are excited in a bounded membrane that acts as a resonant cavity, giving rise to a high-Q response. This results in greater vibrational amplitude for a given input power and greater frequency stability when used in an oscillator circuit. Elimination of the piezoelectric layer simplifies the fabrication and should eventually make FPWs easier to integrate with control electronics. 


\section{DEVICE FABRICATION}

A schematic view of a magnetically-excited FPW (mag-FPW) resonator is shown in Fig. 1. The mag-FPW is formed by (1) depositing low-stress SiN on both sides of a 100 silicon substrate, (2) patterning a rectangular etch mask opening in the backside SiN layer (aligned with the $\langle 110\rangle$ crystal planes of the silicon substrate), (3) patterning a meanderline current conductor on the upper surface (centered with respect to the rectangular SiN opening on the backside), and (4) performing an anisotropic Si etch in $\mathrm{KOH}$ to remove the silicon below the membrane. This leaves a precisely sized, free-standing rectangular membrane held within a silicon frame with current lines positioned to preferentially excite a particular membrane mode.

Fig. 1 Schematic of the magnetically-excited FPW resonator. Excitation of a rectangular membrane mode is achieved by Lorentz forces generated between an alternating current in a meander-line transducer and a static in-plane magnetic field.

\section{PRINCIPLES OF OPERATION}

The operation of the meander-line transducer (MLT) and magnetic field in exciting and detecting FPWs is illustrated in Fig. 1. Current lines, running across the width of the membrane, are positioned at regular intervals along the length of the insulating membrane surface. When a static, in-plane magnetic field is applied perpendicular to the current 
direction, a surface-normal Lorentz stress (force per area) $\vec{F}=\vec{J} \times \vec{B}$ is generated, where $\vec{J}$ is the two-dimensional current density on the membrane surface and $\vec{B}$ is the magnetic flux density. The reversing current direction between meander lines causes alternating surface-normal Lorentz forces. Proper positioning of these lines with respect to the membrane boundaries allows excitation of an eigenmode of the rectangular membrane.

The MLT serves to detect membrane resonances as well as excite them. In a oneport device, motion of the MLT in the magnetic field generates an impedance that can be used to track membrane resonance (an emf is induced due to motion of the conductor in the magnetic field). In a two-port device, the membrane resonance excited by forcing an ac current in a first (input) transducer produces motion that generates a voltage on a second (output) transducer.

Referring to the geometry of Fig. 1, the surface-normal membrane displacement $u$ generated by the Lorentz force is described by the equation ${ }^{4}$ :

$$
D \nabla^{4} u-T \nabla^{2} u+Z_{a} \dot{u}+\rho_{s} \ddot{u}=-B_{x} J_{y}
$$

where $D, T$, and $\rho_{s}$ are the membrane bending moment, tension (force per edge length), and areal mass density, respectively; $Z_{a}$ is a mechanical impedance arising from fluid loading (gas or liquid). Internal membrane damping can be included by using a complex modulus $E^{*}=E(1+j \delta)$, where $\delta$ is the loss tangent, in calculating the bending moment. A rectangular membrane supported at the edges can be excited into a number of 
eigenmodes, with displacement given by ${ }^{4}$ :

$$
u(x, y, t)=A_{m n} \sin \left(k_{m} x\right) \sin \left(k_{n} y\right) e^{j \omega t}
$$

where $A_{m n}$ is the complex displacement amplitude; $k_{m}=m \pi / l$ and $k_{n}=n \pi / w$, where $l$ and $w$ are the membrane length and width, respectively, and $m$ and $n$ are integers; $\omega$ is the angular frequency. Figure 1 shows the membrane being excited into an $(m=6, n=1)$ mode.

Allowing for the possibility of either one or two MLTs on the membrane (one- or two-port devices), the eigenmode amplitude $A_{m n}$ generated is:

$$
A_{m n}=-\frac{2}{j \omega(w l)^{1 / 2}}\left(\frac{K_{1} \hat{I}_{1}+K_{2} \hat{I}_{2}}{Z_{m n}}\right)
$$

where

$$
Z_{m n}=\frac{D k_{m n}^{4}}{j \omega}+\frac{T k_{m n}^{2}}{j \omega}+j \omega \rho_{s}+\frac{D \delta k_{m n}^{4}}{\omega}+Z_{a} .
$$

$\hat{I}_{1}$ and $\hat{I}_{2}$ are the current amplitudes in transducers 1 and 2, while $K$ and $K$ are the respective transducer coupling strengths to the $(m, n)$ membrane mode. (For a one-port device, $K_{2} \hat{I}_{2}=0$.) $Z_{m n}$ is the mechanical impedance (ratio of surface-normal stress to particle velocity) associated with exciting the mode, and $k_{m n}=\left(k_{m}^{2}+k_{n}^{2}\right)^{1 / 2}$. 
Membrane resonances occur at excitation frequencies for which the imaginary part of $Z_{m n}$ vanishes. In a vacuum, where $Z_{a}=0$, this occurs at angular frequencies:

$$
\omega_{m n}=k_{m n}\left(\frac{D k_{m n}^{2}+T}{\rho_{s}}\right)^{\frac{1}{2}}
$$

At resonance, membrane displacement is maximum, limited only by the loss parameters $Z_{a}$ and $\delta$.

As mentioned above, the MLT impresses a surface current density $J_{y}(x, y)$ in a manner designed to preferentially excite a particular membrane mode. The mode coupling factor $K_{i}$ is proportional to the spatial correlation between the current density $J_{y}(x, y)$ and the $(m, n)$ eigenmode displacement:

$$
K_{i}=\frac{2 B_{x}}{(w l)^{1 / 2} \hat{I}_{i}} \int_{0}^{w} \int_{0}^{l} \hat{J}_{y}^{(i)}(x, y) \sin \left(k_{m} x\right) \sin \left(k_{n} y\right) d x d y \cong \frac{8 B_{x} N e}{\pi}\left(\frac{w}{l}\right)^{\frac{1}{2}}
$$

where $\hat{J}_{y}^{(i)}$ is the amplitude of current density applied by the $i^{\text {th }}$ MLT, $N$ is the number of MLT periods, and $e$ is the coupling efficiency $(\leq 1)$. The approximation indicated in the second expression of Eq. 6 is determined by modeling the current lines as infinitesimally wide and positioned along longitudinal mode (index $m$ ) antinodes. This means the meander-line legs are centered at $[\lambda / 4+p(\lambda / 2)]$, where $\lambda$ is the longitudinal mode wavelength (see Fig. 1) and $p$ is an integer. (The coupling efficiency $e$ accounts for the 
finite width and imperfect positioning of the MLT lines that occur in practice.) The MLT then couples preferentially to the desired longitudinal mode with minimal coupling to others having non-matching periodicities.

The voltage $V_{i}=\hat{V}_{i} e^{j \omega t}$ across an MLT has contributions from ohmic resistance in the line and a back-emf due to motion of the conductor in the magnetic field:

$$
\hat{V}_{i}=\hat{I}_{i} R_{o i}-\frac{\partial}{\partial t} \int \vec{B} \cdot d \vec{A}=\hat{I}_{i} R_{o i}+K_{i}\left(\frac{K_{1} \hat{I}_{1}+K_{2} \hat{I}_{2}}{Z_{m n}}\right)
$$

where $i$ indicates the transducer and $R_{o i}$ is the associated ohmic resistance; the integral is over the "coil" area $A$ (with normal $\hat{x}$ ) defined by the displaced MLT lines.

From Eq. 7 , the MLT voltages $\left(V_{1}, V_{2}\right)$ and currents $\left(I_{1}, I_{2}\right)$ near an isolated membrane resonance are related by an impedance matrix:

$$
\left(\begin{array}{c}
\hat{V}_{1} \\
\hat{V}_{2}
\end{array}\right)=\left(\begin{array}{cc}
R_{o I}+\frac{K_{1}^{2}}{Z_{m n}}, & \frac{K_{1} K_{2}}{Z_{m n}} \\
\frac{K_{1} K_{2}}{Z_{m n}}, & R_{o 2}+\frac{K_{2}^{2}}{Z_{m n}}
\end{array}\right)\left(\begin{array}{l}
\hat{I}_{1} \\
\hat{I}_{2}
\end{array}\right) .
$$

The input impedance for the one-port device is then:

$$
Z_{1}(\omega)=\left.\frac{\hat{V}_{1}}{\hat{I}_{1}}\right|_{\hat{I}_{2}=0}=R_{o I}+\frac{K_{1}^{2}}{Z_{m n}}
$$


while the transmission response for the two-port device is given by the forward transmission scattering parameter ${ }^{5}$ :

$$
S_{21}=\frac{2 z_{21}}{\left(z_{11}+1\right)\left(z_{22}+1\right)-z_{12} z_{21}}
$$

where the $z_{i j}$ in Eq. 10 are elements of the $2 \times 2$ impedance matrix in Eq. 8, normalized to $50 \Omega$.

From Eq. 8, the electrical characteristics of one- and two-port mag-FPW resonator near an isolated $(m, 1)$ membrane resonance can be represented by the equivalent-circuit models in Fig. 2. These consist of a parallel LCR resonator that is transformer-coupled to the terminal(s). The parallel membrane elements together represent the mechanical impedance of the membrane mode. From Eq. 4, this impedance has contributions from the membrane bending moment $\left(L_{1}\right)$, tension $\left(L_{2}\right)$, mass density $\left(C_{1}\right)$, internal dissipation $\left(R_{1}\right)$ and fluid loading $\left(L^{\prime}, R^{\prime}\right.$, and $\left.C^{\prime}\right)$. At resonance, the reactive elements (inductances and capacitances) cancel, leaving only a real impedance. Coupling to the membrane mode is represented by an ideal transformer with turns ratio $K_{1}$ that depends (Eq. 6) on the number of MLT periods $(N)$ and magnetic field strength $\left(B_{x}\right)$. The mechanical impedance $Z_{m n}$ (with cgs dimensions $\mathrm{cm}^{2} \mathrm{~s} \mathrm{~g}^{-1}$ ) is transformed (multipled by $K_{1}^{2}$ ) and results in an electrical input impedance $Z_{1}$ (in ohms) that varies with $B_{x}{ }^{2}$. 
When operated in vacuum, the membrane impedance is determined by the "membrane elements" of Fig. 2:

$$
\begin{gathered}
L_{1}=\frac{1}{D k_{m n}^{4}} \\
L_{2}=\frac{1}{T k_{m n}^{2}} \\
C_{1}=\rho_{s} \\
R_{1}=\frac{\omega}{D \delta k_{m n}^{4}} .
\end{gathered}
$$

The mechanical quality factor $(Q)$ of the membrane in vacuum can be calculated from the equivalent circuit ${ }^{6}: Q=\omega R_{1} C_{1}$. When operated in a fluid (gas or liquid), an additional $L^{\prime} R^{\prime} C^{\prime}$ branch arises; these elements will be defined presently.

Fig. 2 Equivalent-circuit models to describe the electrical response of magnetically-excited FPW resonators in the vicinity of a membrane resonance: (a) one-port device, (b) two-port 
device.

Effect of fluid loading Wenzel has shown that FPWs in a membrane generate a flow field (Fig. 3) in a contacting fluid that consists of coupled compressional (surface-normal) and shear (surface-parallel) components ${ }^{7}$. When the FPW velocity is less than the sound velocity in the fluid, this flow field is evanescent, confined to within $\lambda / 2$ of the surface. With a standing FPW, compressional motion dominates above membrane antinodes; above nodes, shear motion prevails.

Fig. 3 The evanescent flow field generated by a standing membrane FPW in a contacting fluid consists of a superposition of surface-normal and in-plane flow.

The mechanical impedance $Z_{\alpha}$ associated with exciting the flow field in a contacting fluid is ${ }^{7}$ :

$$
Z_{a} \cong N_{s} \omega^{2} \rho\left(\frac{s}{k_{m}^{2}-q s}\right)
$$

where $N_{s}$ is the number of membrane sides contacted by the fluid; $q=k_{m}\left[1-\left(v / c_{c}\right)^{2}\right]^{1 / 2}$ and $s=k_{m}\left[1-\left(v / c_{s}\right)^{2}\right]^{1 / 2}$. In the expressions for $q$ and $s, v$ is the FPW propagation velocity; $c_{c}$ and $c_{s}$ are the compressional and shear wave velocities in the fluid. For a Newtonian fluid with density $\rho$ and viscosity $\eta, s \cong[\omega \eta /(2 \rho)]^{1 / 2}(1+j)$ so that $Z_{a}$ can be approximated by series $L^{\prime} R^{\prime} C^{\prime}$ elements defined by: 


$$
\begin{gathered}
R^{\prime}=\omega L^{\prime}=\frac{k_{m}^{2}}{2 N_{s} \omega \rho}\left(\frac{2 \eta}{\omega \rho}\right)^{\frac{1}{2}} \\
C^{\prime}=\frac{N_{s} \rho}{k_{m} \sqrt{1-\left(v / c_{c}\right)^{2}}} .
\end{gathered}
$$

The element $C^{\prime}$ represents energy stored in the evanescent compressional wave and causes a decrease in the resonant frequency of the FPW resonator given by:

$$
\frac{\Delta f}{f_{o}}=-\frac{N_{s} \rho}{2 k_{m} \rho_{s} \sqrt{1-\left(v / c_{c}\right)^{2}}} .
$$

The elements $L^{\prime}$ and $R^{\prime}$ arise from shear wave generation in the fluid. Shear fluid motion gives rise to energy storage $\left(L^{\prime}\right)$, while gradients in the shear displacement lead to power dissipation $\left(R^{\prime}\right)$ in the viscous fluid. A convenient measure of the fluid-induced membrane damping is the inverse motional resistance contributed by the $L^{\prime} R^{\prime} C^{\prime}$ branch at resonance:

$$
\frac{1}{R_{2}}=\frac{N_{s}}{1-\left(v / c_{c}\right)^{2}}\left(\frac{\omega \rho \eta}{2}\right)^{\frac{1}{2}}
$$




\section{RESULTS AND DISCUSSION}

Membrane Properties The areal mass density $\rho_{s}$ of the membrane was estimated from the thicknesses and densities of the SiN layer $\left(h_{1}=2 \mu \mathrm{m}, \rho_{1}=2.95 \mathrm{~g} / \mathrm{cm}^{3}\right)$, Cr metallization layer $\left(h_{2}=0.015 \mu \mathrm{m}, \rho_{2}=7.20 \mathrm{~g} / \mathrm{cm}^{3}\right)$, and Au metallization layer $\left(h_{3}=0.5 \mu \mathrm{m}, \rho_{3}=\right.$ 19.3 $\mathrm{g} / \mathrm{cm}^{3}$ ). With electrodes $\lambda / 4$ wide and positioned at membrane antinodes, the effective areal mass density is estimated at $\rho_{s}=1.39 \mathrm{mg} / \mathrm{cm}^{2}$.

To determine the membrane bending moment $D$ and in-plane tension $T$, FPW resonators with various transducer periodicities were constructed on the same wafer. Fitting Eq. 5 to the $\omega$-vs. $-\mathrm{k}$ data measured for these devices yields $D$ and $T$ for the overall membrane, including $\mathrm{SiN}$ and metallization layers: $D=1.84$ dyne-cm, $T=1.22 \times 10^{5}$ dyne $/ \mathrm{cm}$. The latter leads to an in-plane stress $\left(T / h_{1}\right)$ of $6.10 \times 10^{8} \mathrm{dyne} / \mathrm{cm}^{2}$. Using literature values for the bulk SiN Young's modulus and Poisson's ratio, we calculate the bending moment of the SiN layer alone as $D=1.9$ dyne-cm, which is close to the measured value. In addition, the extracted value of the membrane stress is in reasonable agreement with what has been reported for low-stress silicon nitride films.

One-port resonator response Figure 4 shows the impedance response $Z_{1}(f)$ for a one-port mag-FPW resonator near the $(14,1)$ mode resonance. This device has 7 MLT periods with a periodicity $\lambda=320 \mu \mathrm{m}$. The SiN membrane dimensions are nominally $l=7 \lambda=2.2$ $\mathrm{mm}, w=10 \lambda=3.2 \mathrm{~mm}$, and $h_{1}=2 \mu \mathrm{m}$. The device has a dc resistance $R_{o 1}=24.98$ $\Omega$, with a resonance peak that increases with magnetic field strength. In calculating the 
impedance response (solid line in Fig. 4) near this isolated membrane resonance, we use Eq. 9 with $K_{1}$ given by Eq. 6 and $Z_{m n}$ by Eq. 4 The response is calculated using the measured values of magnetic field strength $\left(B_{x}\right)$ and a constant set of parameters. These include the dc resistance and the membrane parameters $T, D$, and $\rho_{s}$ determined above, along with best-fit values for the mechanical damping parameter $R_{1}=1.094 \mathrm{~cm}^{2} \mathrm{~s} \mathrm{~g}^{-1}$ and coupling efficiency $e=0.26$. The resonant frequency, $f_{o}$, is $409.35 \mathrm{kHz}$. The responses in Fig. 4, measured in vacuum, indicate a membrane (mechanical) $Q$ value of 4300 . Owing to electrical loading by the input resistance $R_{\mathrm{o}}$, the electrical $Q$ is about 50 .

Fig. 4 Impedance response for a single-port mag-FPW resonator for several values of magnetic field strength $B_{x}$ (points are measured, lines are calculated).

Figure 5 shows a plot of the change in the electrical motional resistance $\left[Z_{1}\left(f_{o}\right)\right.$ $\left.R_{\mathrm{o} 1}\right]$ vs. magnetic field strength $B_{x}$. The line is a power-law fit with a slope of 2.02 . From Eq. 9 , the motional resistance is proportional to $K_{1}{ }^{2} / Z_{m n}$, with $Z_{m n}=R_{1}$ at membrane resonance in vacuum. Eq. 6 predicts that $K_{1}{ }^{2}$, and hence motional resistance, varies as $B_{x}^{2}$, which is in good agreement with the experimental result: $B_{x}^{2.02}$.

Fig. 5 Motional resistance vs. magnetic field strength. The power-law fit of 2.02 is in good agreement with the $B_{x}^{2}$ model prediction. 
Two-port resonator response Figure 6 shows the transmission response $\left(S_{21}\right)$ for a twoport mag-FPW resonator operating in vacuum. A dominant resonant peak is observed at $433.16 \mathrm{kHz}$, with a resonant insertion loss of $23.9 \mathrm{~dB}$.

Fig. 6 Transmission response $\left(S_{21}\right)$ for a two-port mag-FPW resonator operating in vacuum.

The device whose response is shown in Fig. 6 has two MLTs, each having 7 periods with a periodicity of $320 \mu \mathrm{m}$. The spacing between MLTs is $1.5 \lambda$. The SiN membrane dimensions are $l=15.5 \lambda=4.96 \mathrm{~mm}, w=10 \lambda=3.2 \mathrm{~mm}$, and $h_{1}=2 \mu \mathrm{m}$. In Fig. 6 the points are measured values while the line is calculated from Eq. $10\left(z_{i j}\right.$ from Eq. $8, K_{i}$ from Eq. 6, and $Z_{m n}$ from Eq. 4) using the measured value of magnetic field strength $\left(B_{x}\right.$ $=4.64 \mathrm{kG}$ ) and the membrane parameters determined above. The MLT parameters determined by fitting the data (measured in vacuum) include $R_{1}=1.90 \mathrm{~cm}^{2} \mathrm{~s} \mathrm{~g}^{-1}, e=$ 0.22 , and $\rho_{s}=1.37 \mathrm{mg} / \mathrm{cm}^{2}$. The membrane $\mathrm{Q}$ for this device operating in vacuum is 7060. The MLT efficiencies for the two-port resonator (22\%) are comparable to those of the one-port resonator $(26 \%)$.

Figure 7 shows the variation in resonant insertion loss with magnetic field strength for this two-port mag-FPW resonator. The points are measured values, while the line is calculated from the model. In this plot, insertion loss (in decibels) varies linearly with $\log \left(B_{x}\right)$ for $B_{x}$ below about $4.3 \mathrm{kG}$. In this regime, insertion loss varies approximately 
$38.7 \mathrm{~dB}$ per decade of magnetic field strength. For weak transducer coupling, the coupled energy into and out of the membrane is expected to vary as $B_{x}^{2}$ (from the one-port model). This would give a transmission variation of $40 \mathrm{~dB}$ per decade of magnetic field strength, close to what is observed. At higher field strengths or coupling levels, the ports load the membrane resonance, and insertion loss decreases less rapidly with increasing field strength. The linear model captures some of this incremental reduction; nonlinear effects associated with amplitude stiffening may account for the remainder.

Fig. 7 Insertion loss at resonance vs. magnetic field strength for a two-port mag-FPW resonator operating in vacuum.

Gas Loading For the $2 \mu \mathrm{m}$-thick SiN membrane used, the FPW velocity $\left(v=1.3 \times 10^{4}\right.$ $\mathrm{cm} / \mathrm{s}$ ) is lower than the sound velocity in the gases tested. This leads to excitation of an evanescent displacement field (Fig. 3) in the gas next to the membrane surfaces. At a resonant frequency of $400 \mathrm{kHz}$ in nitrogen, the decay length is approximately $78 \mu \mathrm{m}$ for this evanescent field.

Figure 8 shows the fractional change in resonant frequency $\left(\Delta f / f_{\mathrm{o}}\right)$ vs. pressure for a one-port mag-FPW resonator in several gases. The decrease in resonant frequency is due mainly to gas entrainment by the evanescent compressional component of displacement. From Eq. 14, this is expected to vary with gas density $\rho$ which is proportional to pressure. 
Fig. 8 Fractional change in resonant frequency $v$ s. pressure for a one-port resonator in several gases, showing the effect of gas entrainment by the evanescent field (Fig. 3).

Figure 9 shows the variation in inverse motional resistance $\left(1 / R_{2}\right)$, a measure of membrane damping, with pressure for several gases. The points are measured; lines are linear least-squares fits to the data. The damping arises from viscous dissipation occurring above membrane antinodes where there is a significant gradient in shear displacement. Eq. 15 predicts damping will vary as $(\rho \eta)^{1 / 2}$, as is found with thickness-shear mode resonators operating in a Newtonian fluid ${ }^{8}$. Since gas density $\rho$ varies with pressure while viscosity $\eta$ is relatively constant over this range of gas pressures, $1 / R_{2}$ varies as the square root of pressure. On average, the calculated damping due to gas loading is about $15 \%$ less than what was measured. This is most likely due to the radiation of compressional waves at the membrane edges; this arises from the finite extent of the membrane and was not included in the damping model.

Fig. 9 Change in inverse motional resistance $\left(1 / R_{2}\right)$ vs. square root of pressure for a oneport resonator in several gases. The $P^{1 / 2}$ dependence arises from viscous damping due to in-plane fluid motion (Fig. 3).

\section{SUMMARY}

Flexural plate wave resonators have been constructed using bulk micromachining techniques to form a free-standing silicon nitride membrane supported by a silicon frame. 
High-order eigenmodes, e.g. $(14,1)$, can be excited in the rectangular membrane using Lorentz forces. These are generated by surface currents defined by a conductor pattern on the membrane surface in combination with a static in-plane magnetic field.

Efficient coupling to a membrane mode occurs when the current lines are positioned at the longitudinal mode antinodes and alternate in direction every half wavelength. This leads to a meander-line transducer geometry. This transducer couples preferentially to a particular longitudinal mode $(m, 1)$.

At resonance, membrane motion becomes significant. MLT movement in the Bfield induces a voltage on the MLT. For a one-port device, this causes an impedance response. For a two-port device, it generates a voltage on the output transducer. These responses have been modeled and the model gives good agreement with experiment in predicting impedance and transmission (2-port device) vs. frequency, magnetic field, and gas pressure.

Several applications are anticipated for the mag-FPW resonator. The low areal mass density of the membrane makes it extremely sensitive to surface mass accumulation. Hence, addition of a chemically sensitive film should allow sensitive chemical detection. Since the limit of detection is determined by the mass sensitivity of frequency $\left(\Delta f / \Delta \rho_{s}\right)$ divided by the frequency uncertainty (oscillator fluctuation), a useful figure of merit ${ }^{9}$ for resonant sensors is $Q\left(\Delta f / \Delta \rho_{s}\right)$. The relatively high $\mathrm{Q}$ of the FPW resonator, combined with its high mass sensitivity, give it a high figure of merit. 
The mag-FPW resonator is also suitable for other sensing applications. The device can be made very sensitive to changes in membrane tension. This leads to nonlinear

(amplitude-stiffened) responses at higher drive levels ${ }^{10}$, but also makes the device sensitive to other environmental influences that affect membrane tension, such as temperature, differential pressure, and strain.

\section{ACKNOWLEDGMENTS}

The authors are grateful to Prof. T. Kinney of Stanford University for providing

low-stress SiN-coated silicon wafers; to J. L. Dohner, T. Hinnerichs, R. W. Cernosek, G. C. Frye, and M. W. Scott of Sandia National Laboratories for helpful discussions; and to K. Rice of Team Specialty Products for mask layout and graphics support. This work was supported by the U.S. Department of Energy under Contract DE-AC04-94AL85000. Sandia is a multiprogram laboratory operated by Sandia Corporation, a Lockheed Martin Company, for the United States Department of Energy.

\section{DISCLAIMER}

This report was prepared as an account of work sponsored by an agency of the United States Government. Neither the United States Government nor any agency thereof, nor any of their employees, makes any warranty, express or implied, or assumes any legal liability or responsibility for the accuracy, completeness, or usefulness of any information, apparatus, product, or process disclosed, or represents that its use would not infringe privately owned rights. Reference herein to any specific commercial product, process, or service by trade name, trademark, manufacturer, or otherwise does not necessarily constitute or imply its endorsement, recommendation, or favoring by the United States Government or any agency thereof. The views and opinions of authors expressed herein do not necessarily state or reflect those of the United States Government or any agency thereof. 


\section{REFERENCES}

1. R. M. White and S. W. Wenzel, Appl. Phys. Lett., 52 (1988) 1653-55.

2. S. W. Wenzel and R. M. White, IEEE Trans. Electron Devices, ED-35, 735 (1988).

3. T. Giesler and J.-U. Meyer, Sensors and Actuators B, 18-19 (1994) 103-106.

4. L. D. Landau and E. M. Lifshitz, Theory of Elasticity, $3^{\text {rd }}$ ed. (Pergamon Press, New York, 1986), Chs. 2 and 3.

5. S. F. Adam, Microwave Theory and Applications (Prentice-Hall, Englewood Cliffs, NJ) p. 89.

6. R. J. Smith, Electronics: Circuits and Devices (Wiley, New York, 1973), p. 271.

7. S. W. Wenzel, “Applications of Ultrasonic Lamb Waves," Ph.D. thesis, Univ. of Calif., Berkeley (1992).

8. S. J. Martin, V. E. Granstaff, and G. C. Frye, Anal. Chem., 63 (1991) 2272-2281.

9. $\quad$ E. Benes, "Comparison Between BAW and SAW Sensor Principles," IEEE Frequency Control Symp., Lake Buena Vista, FL, May 28 - 30, 1997.

10. M. A. Butler, S. J. Martin, J. J. Spates, and M. A. Mitchell, Tech. Dig. 1997 Int. Conf. on Solid-State Sensors and Actuators, Chicago, June 16-19, 1031-34. 


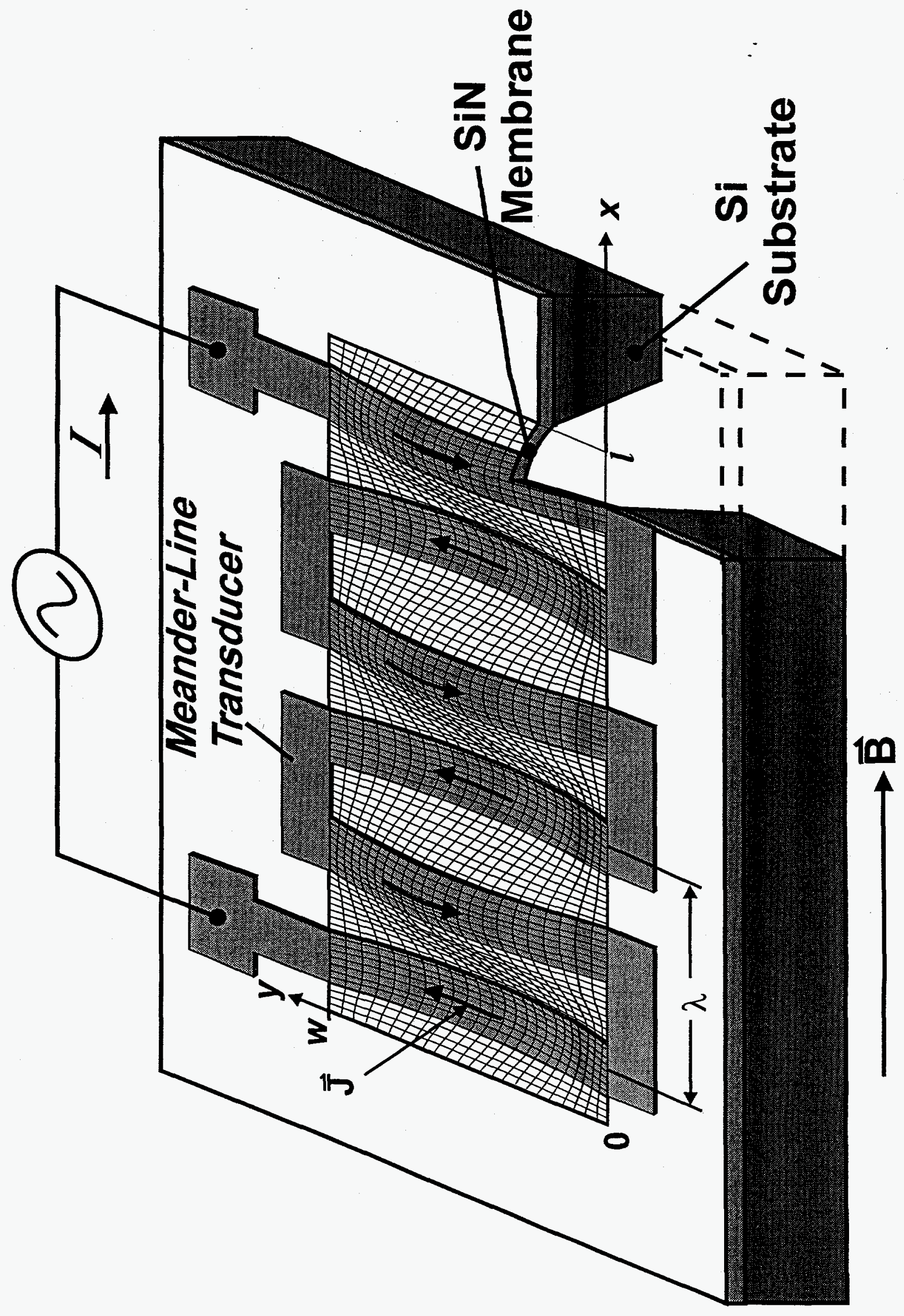




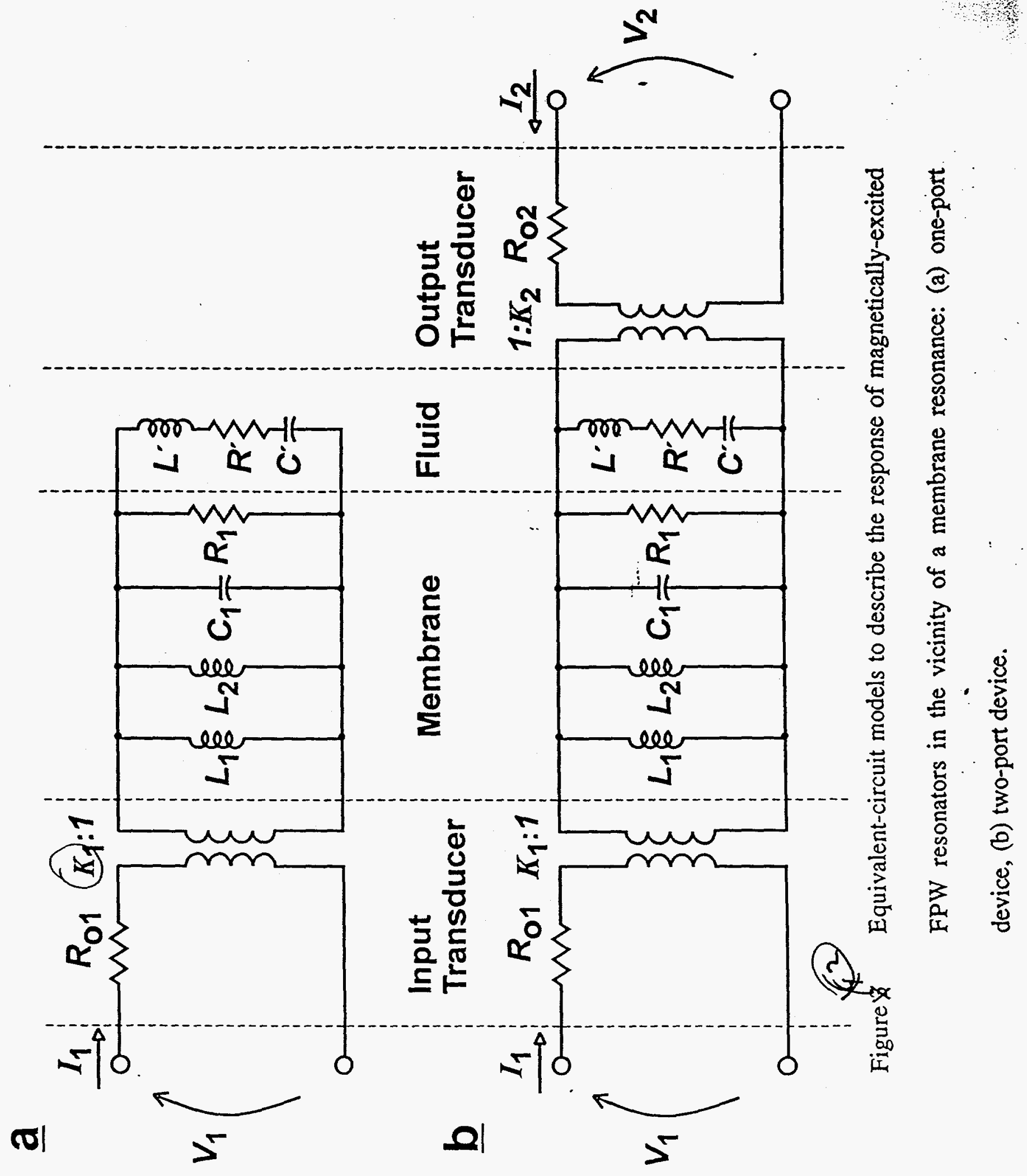




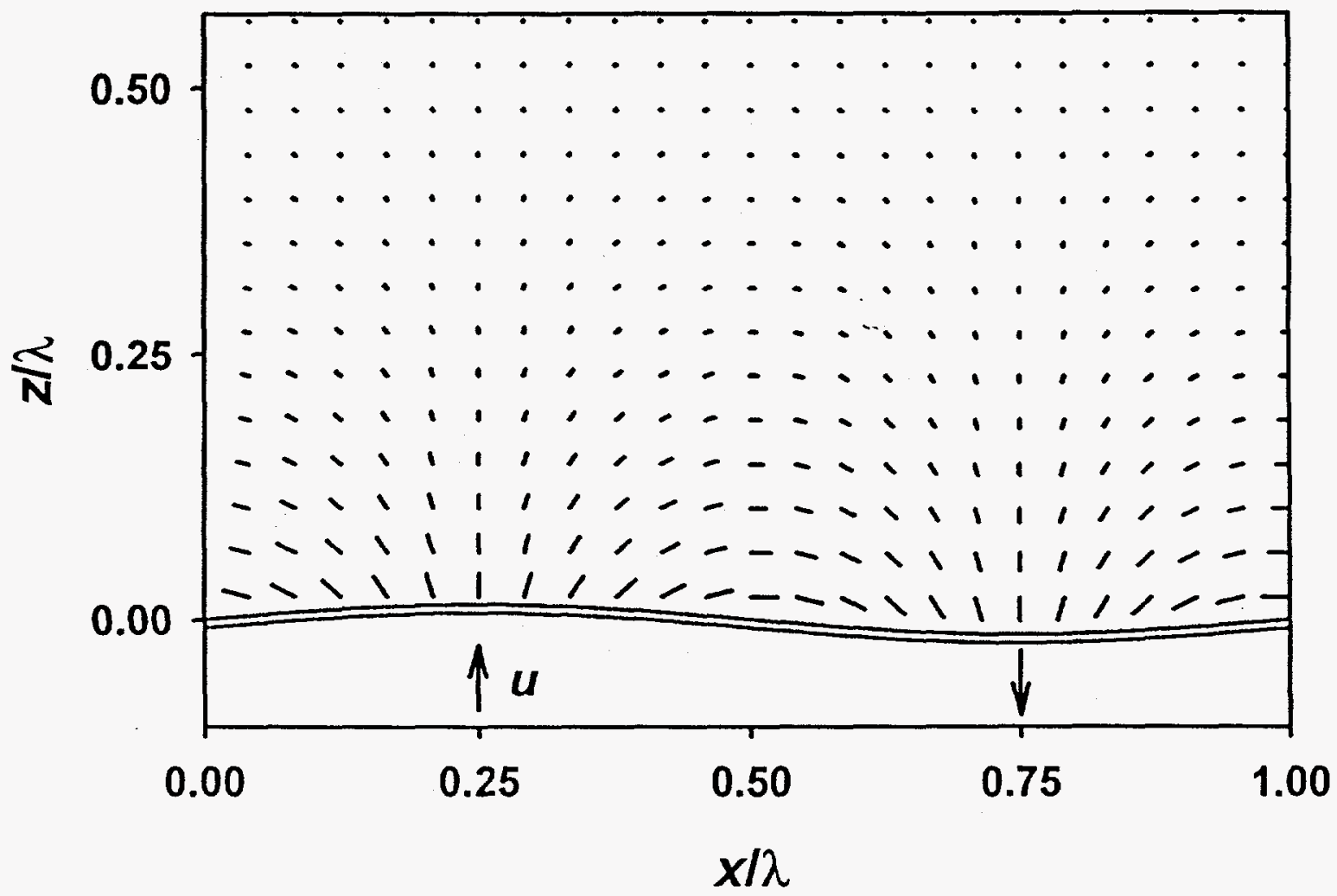

Fig. 3 

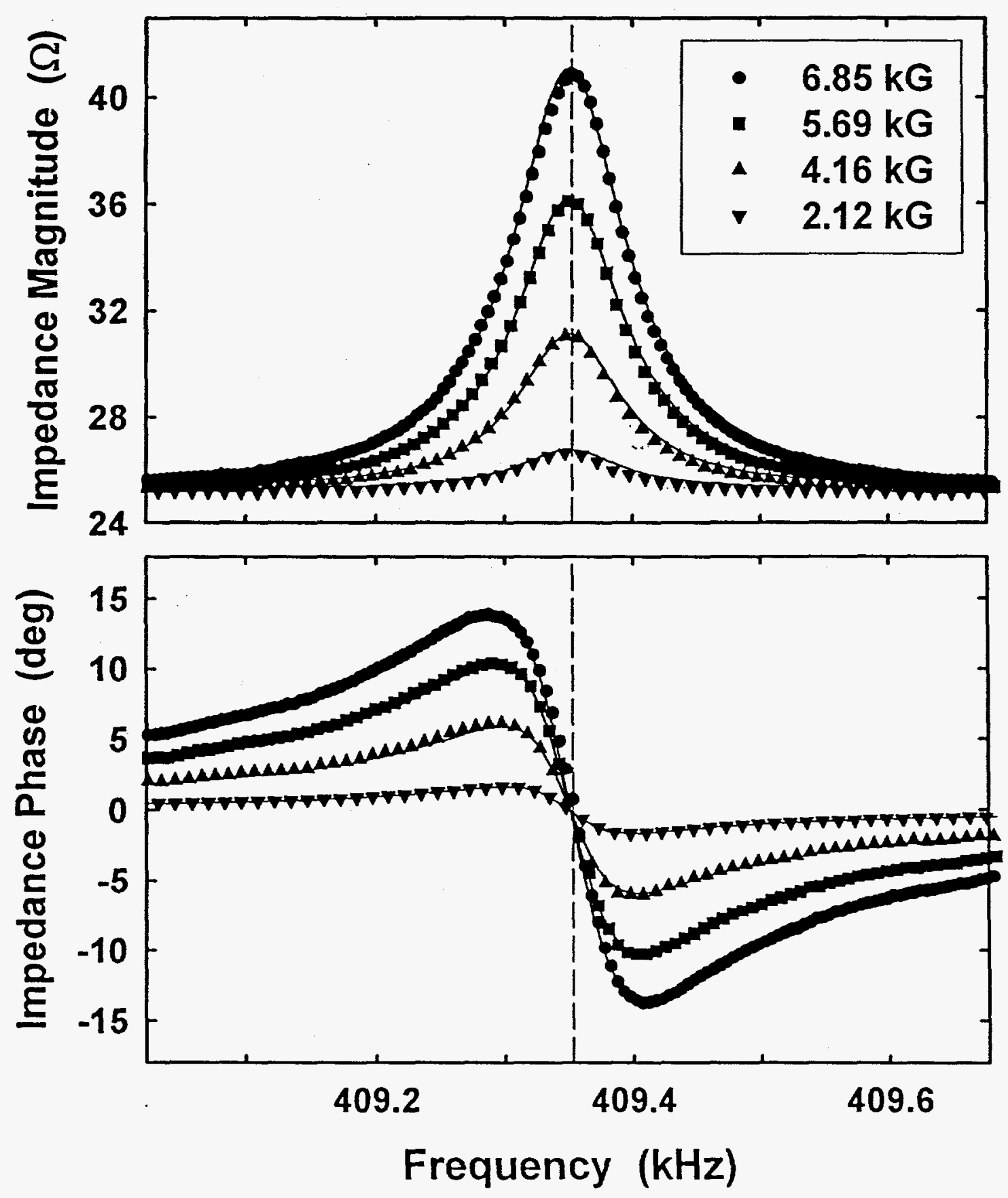

$f_{o}=409.35 \mathrm{kHz}$ 


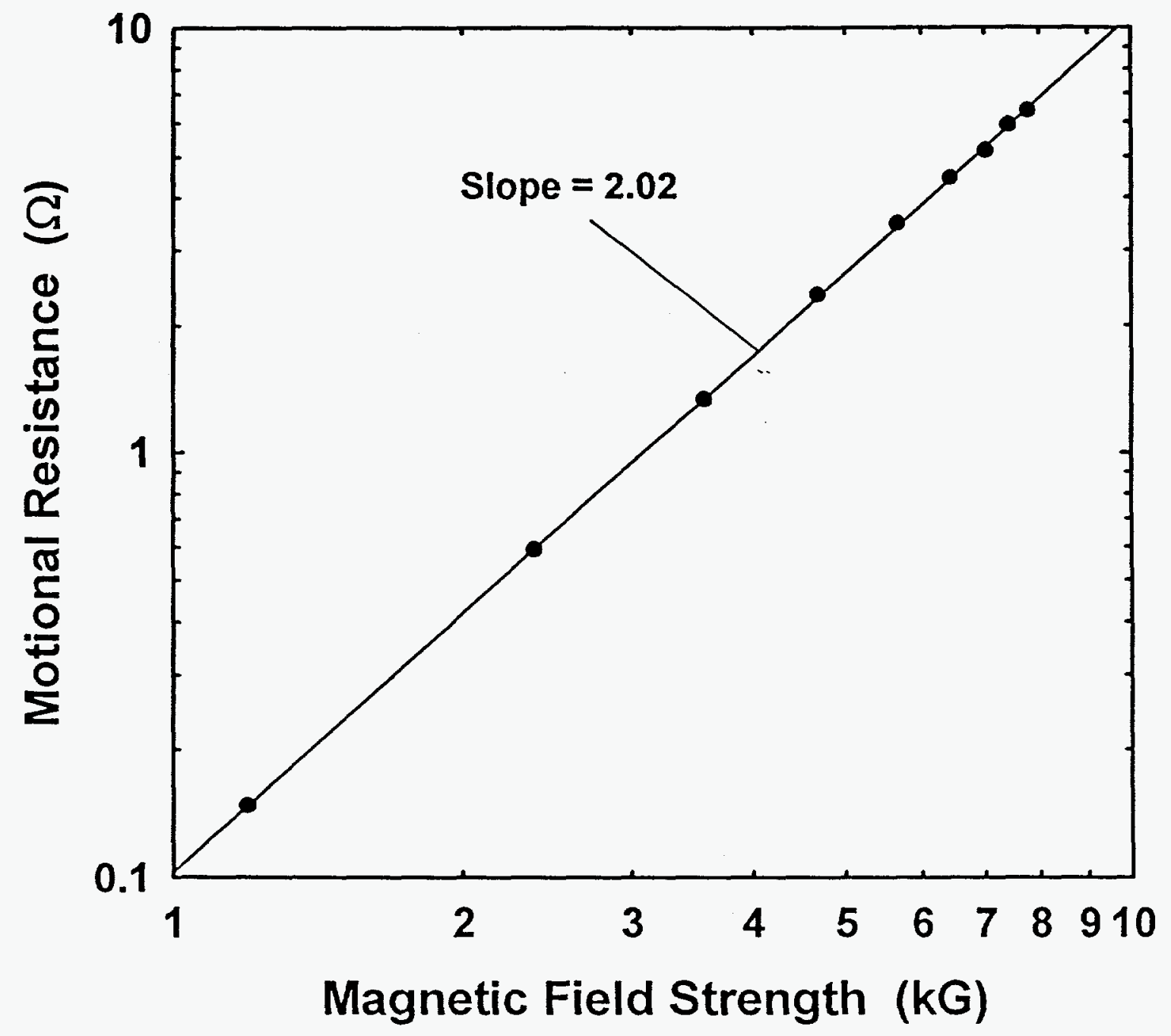

Fig. 5 

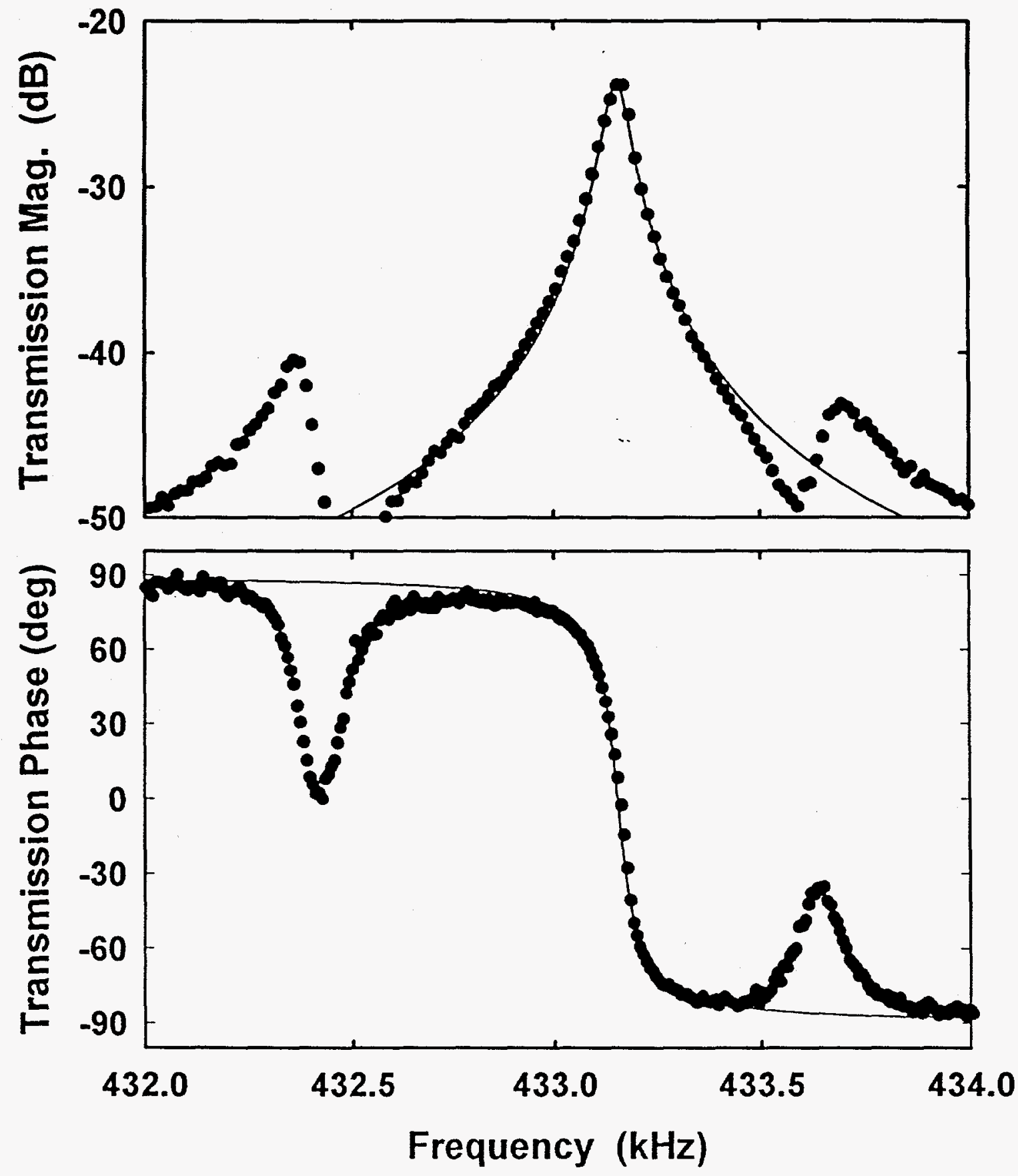

file:Figure 12.jnb

Fog. $\$ 6$ 


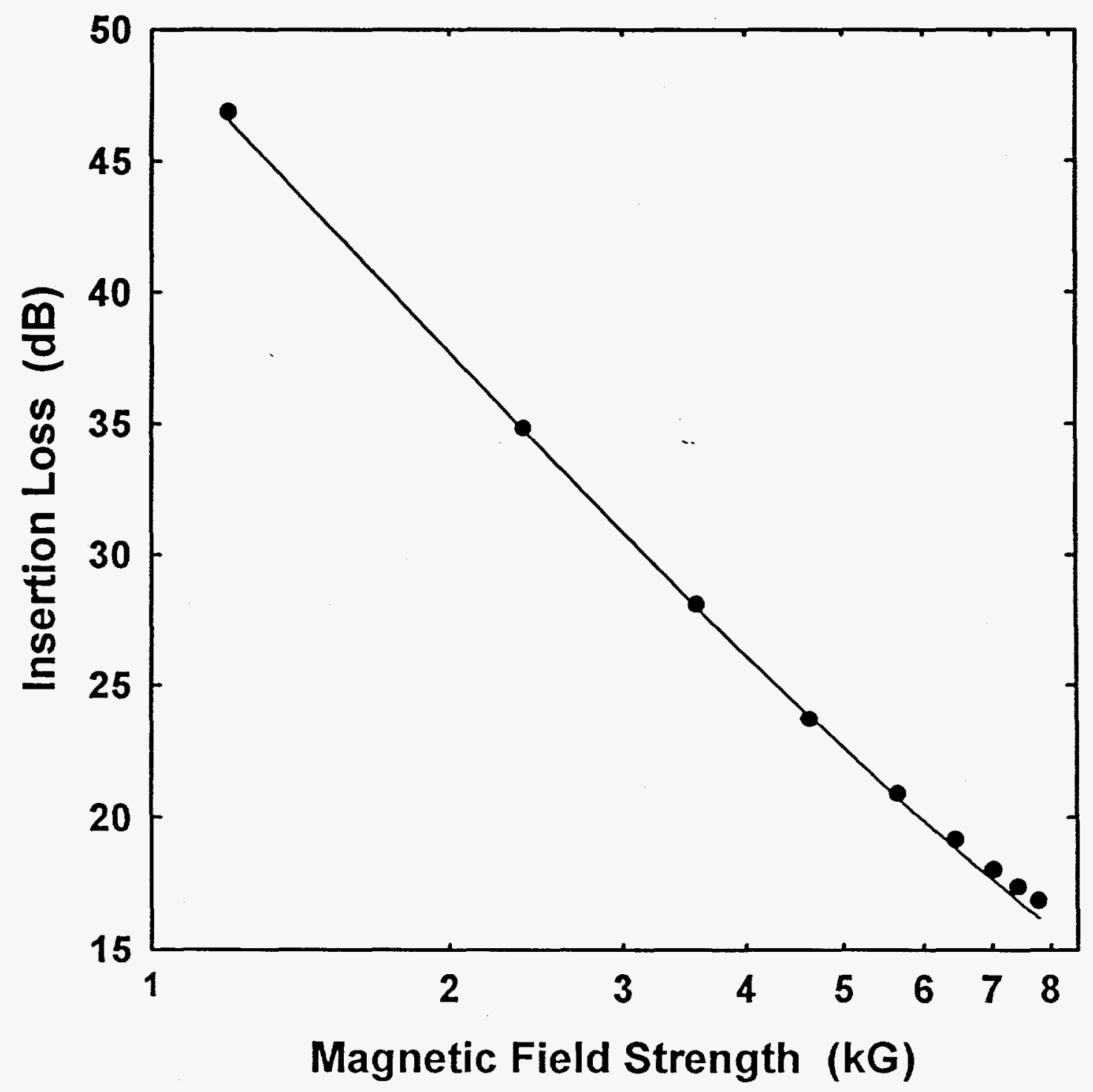

Fg. 1 


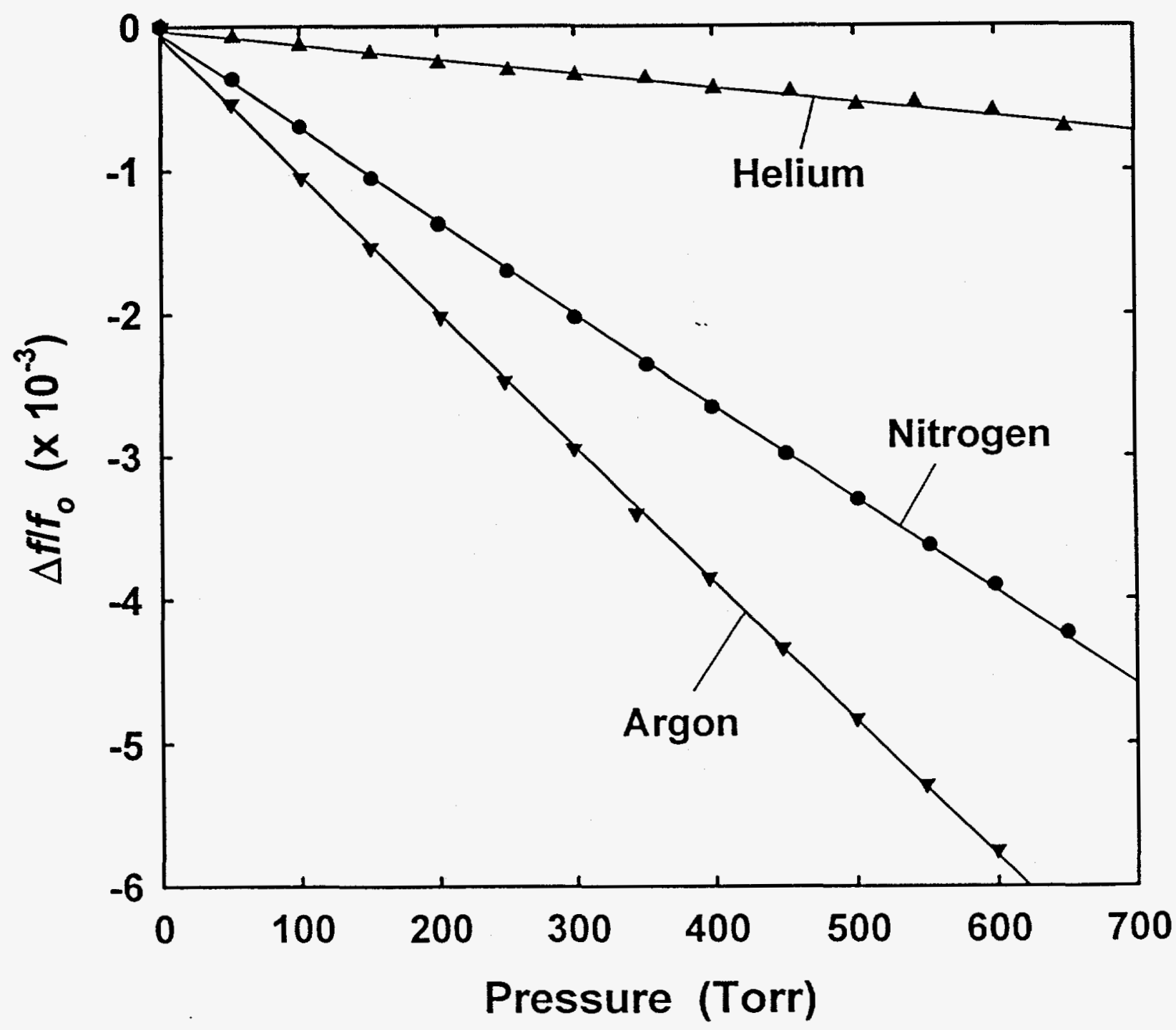

Fig. 8 


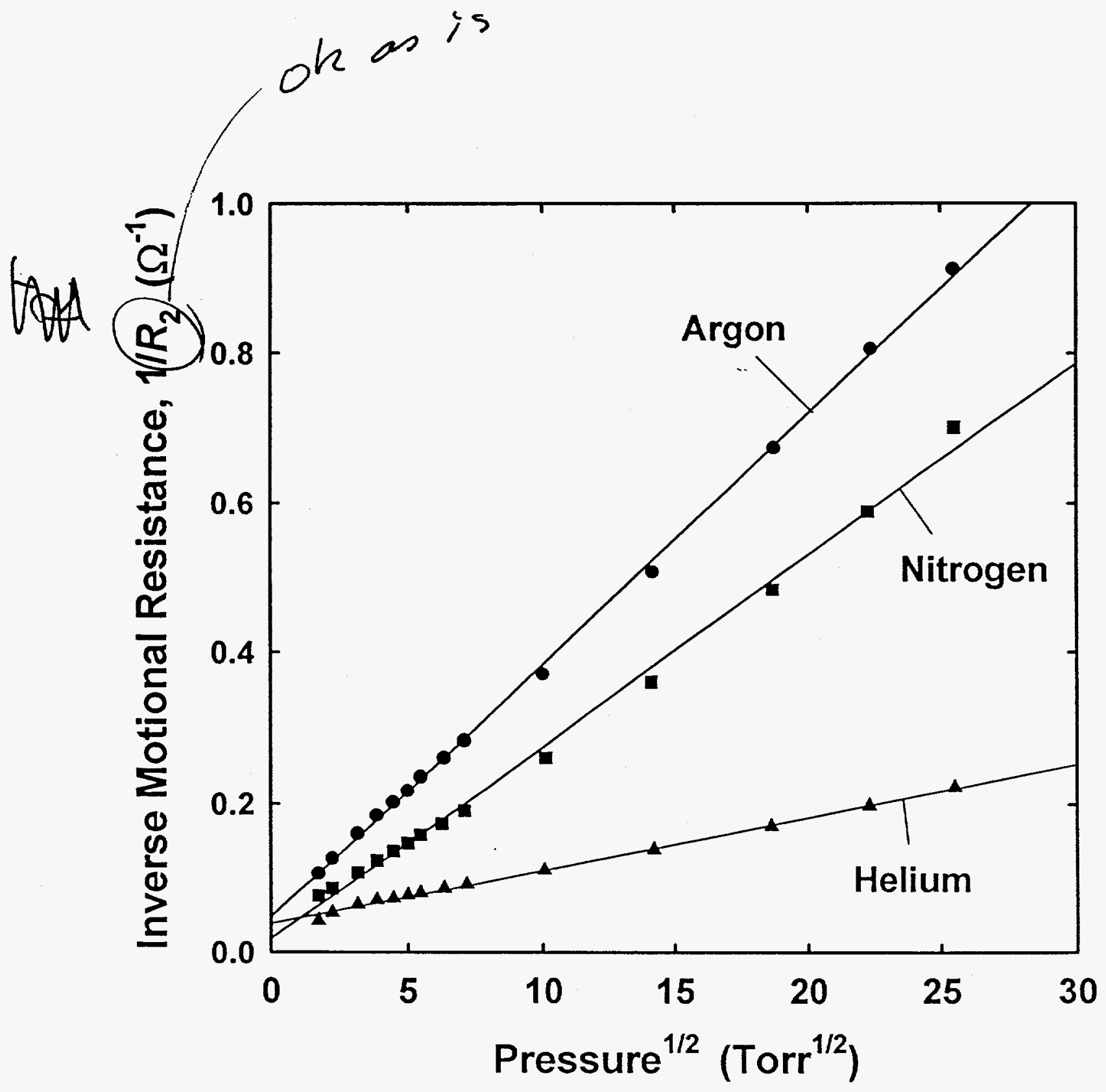

Fig. 9 